Check for updates

Received 4th April 2018

Accepted 16th May 2018

DOI: $10.1039 / c 8 r a 02903 b$

rsc.li/rsc-advances
Cite this: RSC Adv., 2018, 8, 19420

\section{Surface functionality density regulated in situ reduction of nanosilver on hierarchial wrinkled mesoporous silica nanoparticles and their antibacterial activity $\dagger$}

\begin{abstract}
Xuejuan Wan, (D)* Lisi Wu, Hang Pei, Haoqi Ke, Guanghui Yang* and Jiaoning Tang
Hierarchical wrinkled mesoporous silica nanoparticles (WMS NPs) bedecked with diverse functionality density of amino groups (WMSs-N2, WMSs-NN and WMSs-NNN) were first synthesized via typical SolGel method, and then utilized for the in situ reduction of nanosilver with sodium borohydride. Elegantly distributed Ag NPs (ca. 7-10 nm, 3-5 nm) on WMSs-N2 and WMSs-NN without any agglomeration were obtained respectively, while Ag NPs (ca. $50 \mathrm{~nm}$ ) dispersed on WMSs-NNN were obviously larger and slightly agglomerated. Compared to pure Ag NPs, all the obtained Ag@WMSs composites were durable and displayed much better antibacterial performance, with a minimal inhibitory concentration of 12$80 \mathrm{mg} \mathrm{L}^{-1}$ and a minimal bactericidal concentration of $24-108 \mathrm{mg} \mathrm{L}^{-1}$, respectively. Moreover, it was found that the functionality density of amino groups and the specific surface area of WMSs played a crucial role for the antibacterial performance of the obtained nanocomposites. Because WMSs-NN had higher specific surface area and surface amino density than WMSs-N2, the size and dispersion of Ag NPs on WMSs-NN were smaller and superior to those of Ag NPs on WMSs-N2, respectively. Accordingly, Ag@WMSs-NN displayed a better antibacterial capacity than Ag@WMSs-N2. As for Ag@WMSs-NNN, owing to the high loading content of Ag NPs, they exhibited the best antibacterial and bactericidal properties.
\end{abstract}

\section{Introduction}

Bacterial contamination has attracted much attention in food hygiene, environmental safety and public health. ${ }^{1-3}$ Presently, a great deal of research has focused on nanosilver (Ag NPs) based antibacterial agents due to its powerful antibacterial properties at low concentrations and low toxicity. ${ }^{4-6}$ Nevertheless, Ag NPs are easily aggregated during storage like most metal-based nanoparticles, which causes severe chemical property alteration and antibacterial performance deterioration. ${ }^{7}$ In order to prevent Ag NPs from agglomeration, loading of $\mathrm{Ag}$ NPs on carrier materials has been proven to be an efficient method.

Among the large varieties of carrier materials widely used, mesoporous silica (MS) such as MCM-41, SBA-15 and SBA-16 has long been studied as an ideal candidate for the shielding and sustained releasing of their payload owing to its high specific surface areas, long-range ordered pore channel and various pore structures, ${ }^{8-10}$ which is frequently employed in

Shenzhen Key Laboratory of Polymer Science and Technology, College of Materials Science and Engineering, Shenzhen University, Shenzhen 518060, China. E-mail: wanxj@szu.edu.cn; sabinyang@163.com

$\dagger$ Electronic supplementary information (ESI) available. See DOI:

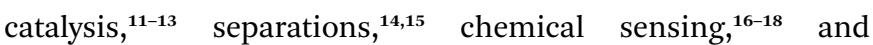
controlled release. ${ }^{19,20}$ Over the past decades, the research efforts on MS-supported Ag NPs are mostly focused on the exploration of appropriate structure regulation of Ag NPs in the MS matrix. ${ }^{21,22}$

During the past decade, numerous amino-functionalized MS framework were reported for the in situ reduction of Ag nanoparticles as amino group can effectively maintain a localized and concentrated silver ion. For example, Tian and coworkers have synthesized Ag NPs decorated MCM-41 nanoparticles by the co-condensation of TEOS and $\mathrm{N}$-(aminoethyl)-amino-propyl trimethoxysilane and proved their enhanced antibacterial effect against both Gram-positive and Gram-negative bacteria. ${ }^{22} \mathrm{Yu}$ et al. have introduced Ag NPs into the hierarchically monolithic silica uniformly using (3-aminopropyl)-triethoxysilane as a modification agent. ${ }^{23}$ Wang has tethered silver ion onto amino-functionalized SBA-15-type MS via the complexing action of $\mathrm{Ag}^{+}-\mathrm{NH}_{2}$, and enhanced antibacterial properties was also obtained..$^{24}$ Although the decoration of Ag NPs on aminofunctionalized MS has been numerously demonstrated, the effects of amino functionality density on the particle size, morphology, the loading efficiency of Ag NPs and their regulation toward the antibacterial performance all stayed unexplored. 
On the other hand, it has been reported that the steady release of silver ions also play a critical role to the antibacterial capacity of the Ag NPs-loaded MS systems, so the structure of MS should be considered when designing and optimizing these systems. ${ }^{22}$ Recently, hierarchical wrinkled mesoporous silica materials (WMSs), a new member of silica frameworks family, have attracted considerable attention in the field of carrier materials. ${ }^{25}$ Compared with traditional MCMs and SBAs with ordered and monomodal mesopores, WMSs present hierarchical pore structures and radially oriented open pores, which makes it a promising substrate for loading Ag NPs as an antibacterial agent. ${ }^{26}$ In fact, we have recently, for the first time, demonstrated that the durability and antibacterial performance of $\mathrm{Ag}$ NPs-loaded WMSs system are much better than that of pure colloidal Ag NPs. ${ }^{27}$ Inspired by this study, we naturally wonder whether the antibacterial ability of Ag NPs-loaded WMSs system can be further improved by regulating the dispersion of Ag NPs.

In this study, Ag NPs are in situ reduced onto different WMSs with diverse amino modification density, while WMSs are synthesized directly by co-condensation of 3-aminopropyltriethoxysilane ( $\mathrm{N}$ silane, APTES), $\mathrm{N}$-(2-aminoethyl)-3aminopropyltrimethoxysilane (NN silane, KH-792), or trimethoxysilyl propyl diethylenetriamine (NNN silane, NQ-62) with tetraethoxysilane (TEOS), respectively (Fig. S1†). Considering that the amino groups in APTES, KH-792 and NQ-62 on WMSs provide different number of active sites for the absorption of $\mathrm{Ag}^{+}$, diverse $\mathrm{Ag}$ NPs loading situation will be obtained. The size and morphology of Ag NPs generated in WMSs-N, WMSs-NN, WMSs-NNN, combined with their antibacterial performance are recorded and compared in detail.

\section{Experimental}

\subsection{Materials and instruments}

TEOS (AR, 28.0\%), APTES (N silane), KH-792 (NN silane), NQ-62 (NNN silane) and CTAB (AR, 99.0\%) were supplied by Sinopharm Chemical Reagent Company. Silver nitrate $\left(\mathrm{AgNO}_{3}, \mathrm{AR}\right.$, 99.8\%), sodium borohydride $\left(\mathrm{NaBH}_{4}, \mathrm{AR}, 98.0 \%\right)$, poly( $N$-vinylpyrrolidone) (PVP-K30, Wt40000) and all the solvents were purchased from Aladdin Industrial Corporation. The detailed information of instruments and characterization process are listed in the ESI. $\dagger$

\subsection{Synthesis of APTES modified WMSs (WMSs-N/WMSs-N2)}

$0.50 \mathrm{~g}$ CTAB was dissolved in $70 \mathrm{~mL}$ water, and then this solution was mixed with $5 \mathrm{~mL}$ ethyl alcohol, $15 \mathrm{~mL}$ diethyl ether and $0.80 \mathrm{~mL}$ ammonium hydroxide. After that, the mixture was stirring mixed with $2.50 \mathrm{~mL}$ TEOS and $0.20 \mathrm{~mL}$ APTES. After $4 \mathrm{~h}$ of stirring, the reaction was stopped by centrifuging the mixture. The obtained precipitate was washed thrice with ethyl alcohol, and subsequently refluxed overnight in a mixture of concentrated $\mathrm{HCl}(15 \mathrm{~mL})$ and ethyl alcohol $(120 \mathrm{~mL})$. Finally, WMSs-N was obtained by centrifuging and washing the precipitate with water.
$1 \mathrm{~g}$ WMSs-N was ultrasonically dispersed in $100 \mathrm{~mL}$ methylbenzene. Then, this dispersion was mixed with 2 mL APTES, and subsequently stirred at $80{ }^{\circ} \mathrm{C}$ for $18 \mathrm{~h}$. After that, the mixture was centrifugated and washed with water. Finally, WMSs-N2 was obtained by vacuum drying the obtained precipitate at $60{ }^{\circ} \mathrm{C}$.

\subsection{Synthesis of KH-792 modified WMSs (WMSs-NN) and NQ-62 modified WMSs (WMSs-NNN)}

General procedures for the synthesis of WMSs-NN and WMSsNNN was similar with those of WMSs-N, while $0.2 \mathrm{~mL} \mathrm{KH-792}$ or $0.2 \mathrm{~mL}$ NQ-62 was used as nitrogen-containing siloxane coupling agent instead of APTES.

\subsection{In situ reduction of monodisperse Ag NPs on WMSs}

$80 \mathrm{mg}$ amino-functionalized silica (WMSs-N, WMSs-N2 WMSsNN or WMSs-NNN) was ultrasonically dispersed in $10 \mathrm{~mL}$ ethyl alcohol, and then mixed with $10 \mathrm{~mL} \mathrm{AgNO}$ aqueous solution $\left(1.44 \mathrm{mg} \mathrm{mL}^{-1}\right)$. Thereafter, the electrostatic adherence reaction was started by stirring the mixture under dark. After $80 \mathrm{~min}, 8 \mathrm{mg}$ PVP-K30 in $5 \mathrm{~mL}$ water was added, and subsequently added dropwise with $5 \mathrm{~mL} \mathrm{NaBH}_{4}$ aqueous solution $\left(0.8 \mathrm{mg} \mathrm{mL}{ }^{-1}\right)$. After magnetic stirring for another $15 \mathrm{~min}$, the reaction was by centrifuging the mixture. The obtained precipitate was washed with water and ethyl alcohol, followed by freeze-drying for $15 \mathrm{~h}$. The obtained samples were designated as Ag@WMSs-N, Ag@WMSs-N2,Ag@WMSs-NN, and Ag@WMSsNNN, respectively.

\subsection{Evaluation of antibacterial properties}

Minimal inhibition concentration (MIC) test. Fresh bacteria were inoculated in liquid medium and grown to an approximate OD600 of 0.5 (i.e. the initial concentration is about $1 \times 10^{8} \mathrm{CFU}$ $\left.\mathrm{mL}^{-1}\right)$. After that, the suspension was diluted to $1 \times 10^{6} \mathrm{CFU}$ $\mathrm{mL}^{-1}$, and followed by adding with different contents of samples (Ag@WMSs-N2, Ag@WMSs-NN, Ag@WMSs-NNN). MIC was tested after cultivating the mixed suspension in an orbital shaker at $37{ }^{\circ} \mathrm{C}$ for $24 \mathrm{~h}$. The experiment included a positive control (flask containing Ag@WMSs and nutrient media, without inoculum) and a negative control (flask containing inoculums and nutrient media, without Ag NPs). The absorbance values for experimental flasks were corrected by deducting the corresponding absorbance values for the positive controls.

Minimal bactericidal concentration (MBC) test. $0.1 \mathrm{~mL}$ of the above cultivated sterile suspension was extracted. The extractive was spread on solid medium and cultivated for another $24 \mathrm{~h}$. The average number of discrete colonies was counted as the number of the remaining bacteria by repeating the above procedure three times with error bars. The minimal concentration of samples (Ag@WMSs-N2,Ag@WMSs-NN, Ag@WMSsNNN) at which the bacteria growth rate reduced more than $99 \%$ was defined as MBC. 


\section{Results and discussion}

The particle size and morphology of WMSs-N was studied by SEM, HRTEM (Fig. 1a-b) and nitrogen adsorption-desorption (Fig. S2 $\dagger$ ), respectively. It is clear that WMSs-N displayed a spherical wrinkled mesoporous structure with a diameter of $300-350 \mathrm{~nm}$ and radial pore channels. Its BET surface was $602.32 \mathrm{~g} \mathrm{~m}^{-2}$, and the single point adsorption total pore volume is $0.91 \mathrm{~cm}^{3} \mathrm{~g}^{-1}$ (Table S1†). The pore size distribution is consisted with a wide band extending from 10-100 nm and a sharp peak in the range of 3-5 $\mathrm{nm}$, which was similar with the reported literatures. However, $\mathrm{after}^{\mathrm{Ag}}{ }^{+}$ions were adsorbed onto WMSs-N and treated with $\mathrm{NaBH}_{4}$, there was practically no $\mathrm{Ag}$ NPs attaching onto WMSs-N (Fig. 1c, d and S3†). This was probably because a part of the amino groups was buried in the WMSs-N framework in the co-condensation process, leading to an ultra low surface amino density on WMSs-N (as shown in Table $\mathrm{S} 1 \dagger$ ), which ultimately caused a poor in situ Ag nucleation efficiency. ${ }^{28}$

As silanol groups was beneficial to the introduction of functional groups, post grafting of APTES on WMSs-N was then conducted, and the obtained product was denoted as WMSs-N2. In comparison, amino-rich silicone coupling agent $\mathrm{KH}-792$ and NQ-62 was also utilized as the co-condensation agent in the preparation process of WMSs, and the product was denoted as WMSs-NN and WMSs-NNN, respectively. SEM and HRTEM measurement of WMSs-N2, WMSs-NN and WMSs-NNN were shown in Fig. 2. It is clear that the particle morphology of WMSs-N2 was well-remained after the post grafting surface modification, with a slightly decreased BET surface (525.98 g $\mathrm{m}^{-2}$ ). The morphology and the mesopore structure of WMSsNN was quite similar with those of WMSs-N, however, the particle size of it was quite smaller (100-200 nm). The BET surface area and the pore volume of WMSs-NN were $768.83 \mathrm{~g}$ $\mathrm{m}^{-2}$ and $1.03 \mathrm{~cm}^{3} \mathrm{~g}^{-1}$, respectively (Fig. S4 and Table S1 $\dagger$ ). As for WMSs-NNN, the situation was totally different. Larger particle diameter $(550-650 \mathrm{~nm})$ with a much smaller BET surface and pore volume of $241.83 \mathrm{~g} \mathrm{~m}^{-2}$ and $0.23 \mathrm{~cm}^{3} \mathrm{~g}^{-1}$

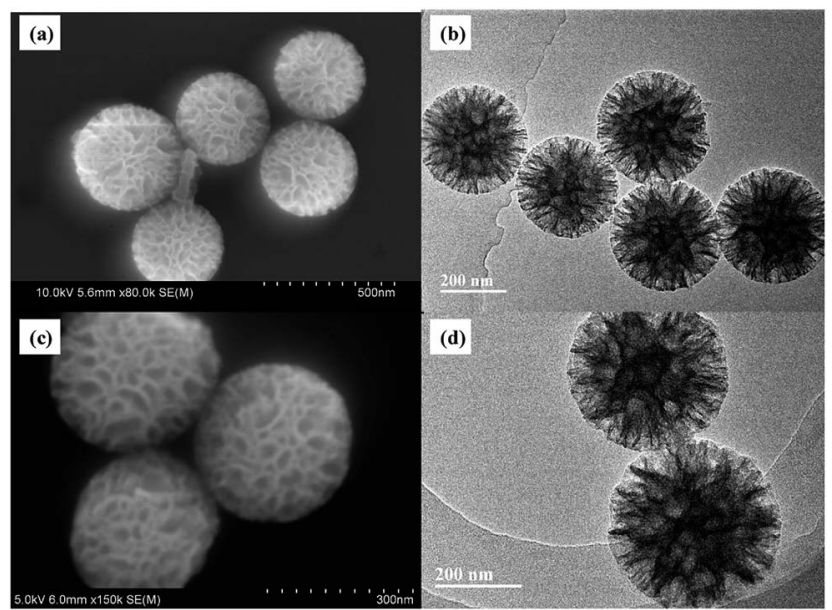

Fig. 1 SEM and HRTEM images of WMSs-N ( $a$ and $b$ ), and Ag@WMSs- $N$ (c and d).
(Fig. S5 and Table S1 $\dagger$ ) were obtained. This phenomenon may attribute to the long siloxane substituent group of NQ-62 and the subsequently disturbed homogeneous configuration of the WMSs-NNN framework. ${ }^{28}$

Under the same experimental conditions, $\mathrm{Ag}^{+}$ions were adsorbed onto these WMSs and in situ reduced with $\mathrm{NaBH}_{4}$. SEM and TEM images of the obtained Ag@WMSs-N2, Ag@WMSs-NN and Ag@WMSs-NNN were shown in Fig. 3. It was obvious that Ag NPs distributed densely on WMSs-N2 and WMSs-NN, with particle size of 7-10 $\mathrm{nm}$ and 3-5 $\mathrm{nm}$ respectively, and no agglomeration was observed. However, the Ag NPs attaching on WMSs-NNN was quite large, showing a diameter of about $50 \mathrm{~nm}$. This phenomenon can probably be attributed to the density difference of amino groups on the surface of WMSs (Table S1 $\dagger$ ). Taking advantages of the high specific surface area, WMSs-N2 and WMSs-NN possessed abundant active amino functional groups on their surface. In the in situ reduction process, these uniformly and densely distributed $\mathrm{Ag}^{+}$-binding sites would provide a great convenience for the formation of a large quantity of $\mathrm{Ag}$ nuclei. Under this circumstance, $\mathrm{Ag}^{+}$ would be suddenly consumed during the nucleation process, which would definitely impact the further growth of the existent nucleus. ${ }^{24,29,30}$ Thus, Ag NPs with relatively small size was formed on WMSs-N2 and WMSs-NN. Compared to WMSs-N2, WMSs-NN had a greater surface amino density, which meant that there were more active sites on WMSs-NN for the formation of $\mathrm{Ag}^{+}-\mathrm{NH}_{2}$ complexing action than that on WMSs-N2. Accordingly, WMSs-NN could form more Ag nucleis than WMSs-N2 in the initial reduction reaction. Since the initial concentration of silver ions in the solution was the same, more Ag nucleis meant the formation of smaller Ag NPs. As for WMSs-NNN, due to its relatively low surface amino density (Table S1 $\dagger$ ), only a small amount of Ag nucleis could be formed in the initial reduction reaction. Therefore, more silver ions would be involved in the further growth of the existent nucleus, leading to the formation of relatively large Ag NPs on WMSs-NNN. It should be noted that the polyamino in silanes NQ-62 played a decisive role for the success in situ loading of Ag NPs on WMSs-NNN. Similar to KH792, the diethylenetriamine functional groups in NQ-62 would effectively enhance the $\mathrm{Ag}^{+}$-capture capacity of the WMSs, thus the Ag NPs loading efficiency of WMSs-NNN was significantly higher than that of WMSs-N.

The existential form of $\mathrm{Ag}$ element in Ag@WMSs-N2, Ag@WMSs-NN and Ag@WMSs-NNN was further investigated by XRD (Fig. S6-S8 $\dagger$ ). The $2 \theta$ peaks at $38.12^{\circ}, 44.30^{\circ}, 64.45^{\circ}$ and $77.41^{\circ}$ could be indexed to the (111), (200), (220) and (311) crystal planes of Ag (JCPDS card no. 04-0783), respectively. Furthermore, there was no peaks of any other silver compounds could be found in all the three compounds. These characterizations suggested that the Ag element in Ag@WMSs was existed in the form of metallic silver. In order to determine the loading content of Ag NPs, the Ag@WMSs were first digested with $\mathrm{HNO}_{3}$, and followed by an ICP-MS measurement. After three parallel tests, the average $\mathrm{Ag}^{+}$concentration was calculated to be $7.68 \mathrm{mg} \mathrm{L}^{-1}, 8.26 \mathrm{mg} \mathrm{L}^{-1}$ and $23.67 \mathrm{mg} \mathrm{L}^{-1}$ based on the standard curves. As the total testing concentration of the abovementioned Ag@WMSs was $125 \mathrm{mg} \mathrm{L^{-1 }}$, the weight 

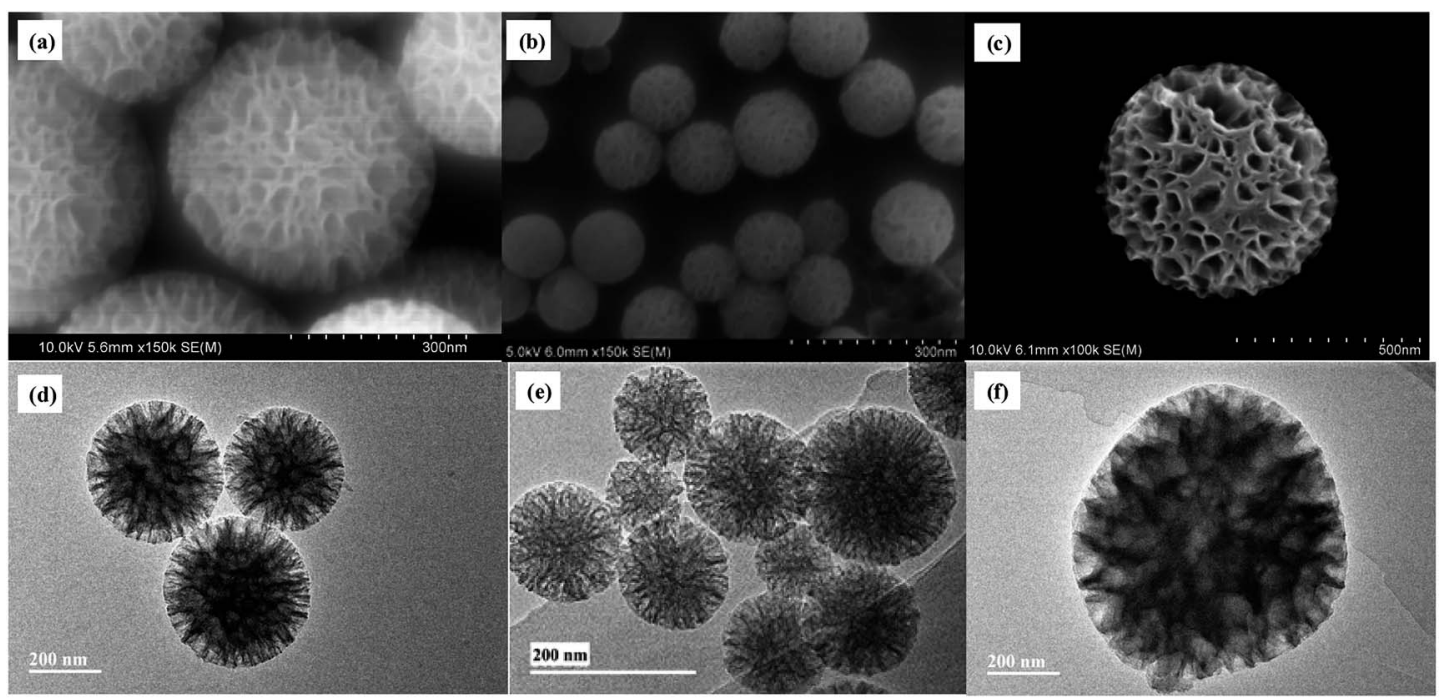

Fig. 2 SEM and HRTEM images of WMSs-N2 ( $a$ and $d$ ), WMSs-NN ( $b$ and e) and WMSs-NNN (c and $f$ ).

percent of Ag NPs on WMSs-N2, WMSs-NN and WMSs-NNN was calculated to be $6.14 \%, 6.61 \%$ and $18.94 \%$, respectively.

Fig. 4 and $\mathrm{S} 9 \dagger$ show the minimal inhibition concentration (MIC) measurement of Ag@WMSs-N2, Ag@WMSs-NN and Ag@WMSs-NNN against Gram-negative bacteria Escherichia coli (E. coli 8099) and Gram-positive bacteria Staphylococcus aureus (S. aureus ATCC 6538). For this study, series concentrations of Ag@WMSs were first cultured with bacterial suspension, and the OD600 values of the suspension were then continuously measured. MIC was defined as the concentration where the OD600 value remained unchanged after cultivating for $24 \mathrm{~h}$. As shown in Fig. 4a and S9a, $\uparrow$ the MIC of Ag@WMSs-N2 for E. coli was $40 \mathrm{mg} \mathrm{L}^{-1}$, while its MIC for $S$. aureus was $80 \mathrm{mg} \mathrm{L}^{-1}$. The higher MIC for $S$. aureus could be ascribed to its thicker cell wall which resulted in higher dose of the antibacterial agent for inhibiting the growth of $S$. aureus. Likewise, the MIC of Ag@WMSs-NN were $24 \mathrm{mg} \mathrm{L}^{-1}$ and $48 \mathrm{mg} \mathrm{L}^{-1}$ for $E$. coli and $S$. aureus (Fig. 4b and S9b $\dagger$ ), respectively. The MIC of Ag@WMSs-
NNN was $12 \mathrm{mg} \mathrm{L}^{-1}$ and $18 \mathrm{mg} \mathrm{L}^{-1}$ for E. coli and $S$. aureus, respectively (Fig. $4 \mathrm{c}$ and $\mathrm{S} 9 \mathrm{c} \dagger$ ).

It should be noted that the MIC of Ag@WMSs prepared in this work is much lower than that of most reported mesoporous silica-supported Ag antibacterial agents (typical 100$1000 \mathrm{mg} \mathrm{L}^{-1}$, Table S2 $\left.\dagger\right),{ }^{22,24,31,32}$ especially for Ag@WMSs-NNN. Moreover, as revealed by ICP-MS measurement, the weight percent of Ag NPs on Ag@WMSs-N2, Ag@WMSs-NN and Ag@WMSs-NNN was 6.14\%, 6.61\% and 18.94\%, thus the actual MIC of Ag NPs on these WMSs carriers were calculated to be 1$2 \mathrm{mg} \mathrm{L}^{-1}$ and 3-4 $\mathrm{mg} \mathrm{L}^{-1}$ for $E$. coli and $S$. aureus, respectively. Compared with the pure Ag NPs with similar size in literature (diameter $\sim 7 \mathrm{~nm}$, MIC is $6.25 \mathrm{mg} \mathrm{L}^{-1}$ and $12.05 \mathrm{mg} \mathrm{L}^{-1}$ for $E$. coli and $S$. aureus, respectively), the Ag NPs in this work displayed much lower MIC.

Standard plate count method was used to visually study the bactericidal activity of Ag@WMSs-N2, Ag@WMSs-NN and Ag@WMSs-NNN (Fig. 5-7 and Table S2 $\dagger$ ). As shown in Fig. 5,

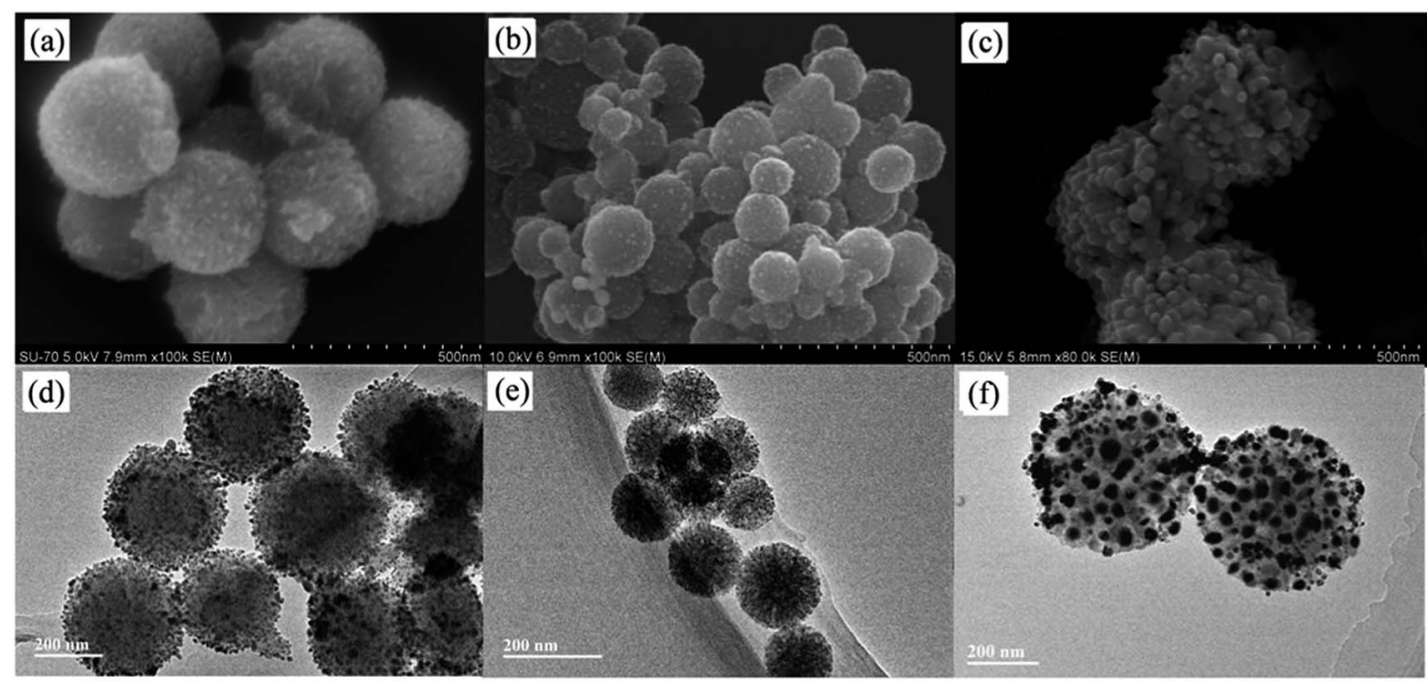

Fig. 3 SEM and HRTEM images of Ag@WMSs-N2 ( $a$ and d), Ag@WMSs-NN (b and e) and Ag@WMSs-NNN (c and f). 

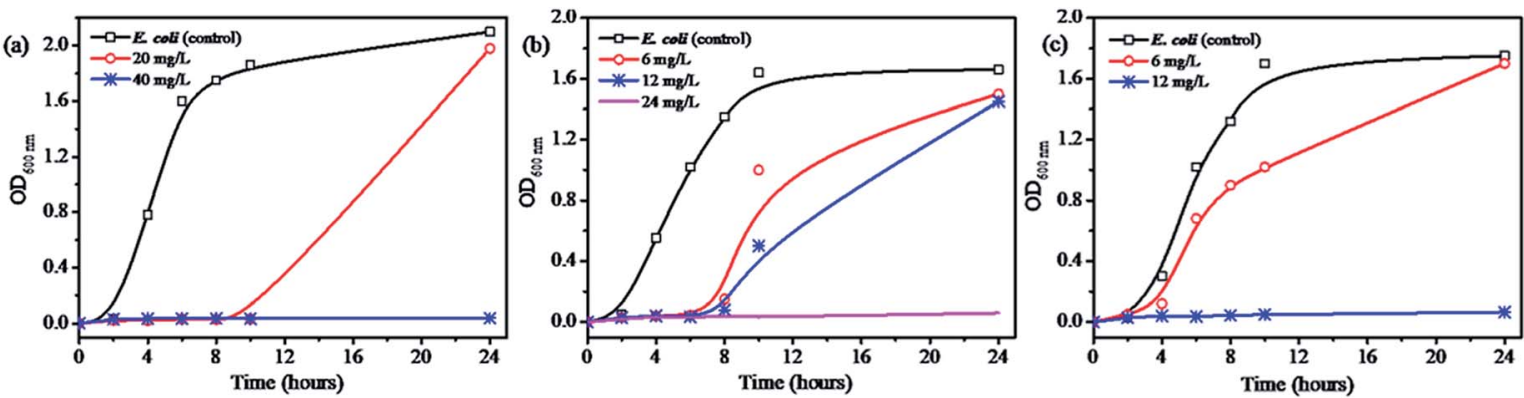

Fig. 4 Concentration effects for the bacterial growth with the addition of Ag@WMSs-N2 (a), Ag@WMSs-NN (b) and Ag@WMSs-NNN (c) toward E. coli.

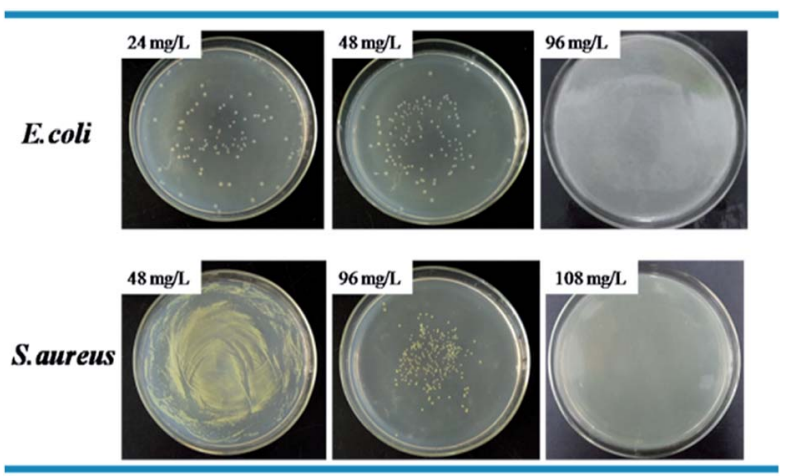

Fig. 5 Bactericidal activity of AgaWMSs-N2 towards E. coli and S. aureus.

MBC of Ag@WMSs-N2 were $96 \mathrm{mg} \mathrm{L}^{-1}$ and $108 \mathrm{mg} \mathrm{L}^{-1}$ for E. coli and $S$. aureus, respectively. Similarly, the MBC of Ag@WMSs-NN were $48 \mathrm{mg} \mathrm{L}^{-1}$ and $96 \mathrm{mg} \mathrm{L}^{-1}$ for $E$. coli and $S$. aureus, respectively (Fig. 6). The MBC of Ag@WMSs-NNN were the lowest, which were $24 \mathrm{mg} \mathrm{L}^{-1}$ and $36 \mathrm{mg} \mathrm{L}^{-1}$ for $E$. coli and $S$. aureus, respectively (Fig. 7). According to the ICP-MS results, the actual MBC of Ag NPs loaded on these three Ag@WMSs samples were calculated to be $3-5 \mathrm{mg} \mathrm{L}^{-1}$ and $6-7 \mathrm{mg} \mathrm{L}^{-1}$ for $E$. coli and $S$. aureus, respectively.

The above results suggested that all the Ag@WMSs obtained in this work showed excellent antibacterial performance against $E$. coli and $S$. aureus. It was speculated that the novel hierarchical morphology and good Ag-loading capacity of mesoporous

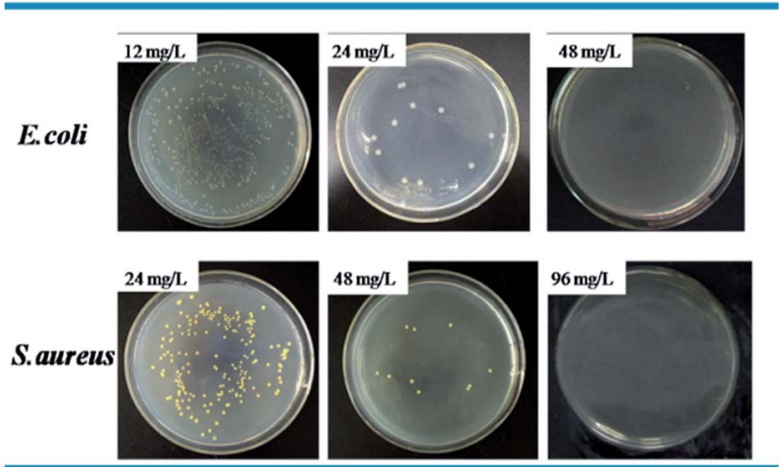

Fig. 6 Bactericidal activity of Ag@WMSs-NN towards E. coli and S. aureus.

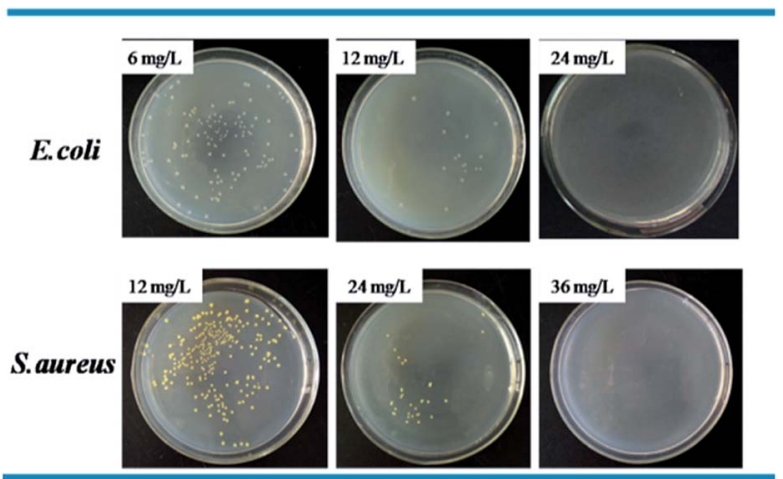

Fig. 7 Bactericidal activity of AgaWMSs-NNN towards E. coli and S. aureus.

silica, and high specific surface area and good dispersion stability of nanoscale Ag particles might all played key roles for the excellent antibacterial performance. As literature reported, Ag NPs could be uniformly and densely loaded on hierarchical structured WMSs. Meanwhile, the center-radial open pores of WMSs could not only protect Ag NPs from deterioration and agglomeration, but also harmonize the dissolution and diffusion of $\mathrm{Ag}^{+}$from Ag NPs. For Ag@WMSs-N2 and Ag@WMSs-NN, though they possessed similar silver content $(6.14 \%$ and $6.61 \%$, respectively), the decorated Ag NPs on WMSs-NN were much smaller than that on WMSs-N2. Agnihotri et al. demonstrated that the bacteriostatic effect of Ag NPs was size and dosedependent, and the smaller the size of the Ag NPs, the better antibacterial property can be obtained. ${ }^{33}$ Thus, Ag@WMSs-NN reasonably displayed better antibacterial properties than Ag@WMSs-N2. As for Ag@WMSs-NNN, the particle size of Ag NPs was quite large, and the dispersibility was slightly inferior to Ag@WMSs-NN. However, the Ag NPs-loading content of Ag@WMSs-NNN (18.94\%) was far greater than the other two samples. Under this situation, the higher Ag NPs loading content may play a decisive role for the excellent antibacterial performance.

\section{Conclusions}

In conclusion, hierarchical wrinkled mesoporous silica (WMSs) with different density of amino groups were synthesized via the co-condensation of APTES, KH-792 or NQ-62 with silane TEOS 
(WMSs-N2, WMSs-NN and WMSs-NNN). Ag NPs with diverse particle size were then densely attached onto the abovementioned silica support via the in situ reduction process, and their loading content was also tested. The obtained Ag@WMSsN2, Ag@WMSs-NN and Ag@WMSs-NNN exhibited a superior antibacterial performance to both $E$. coli and $S$. aureus, with a MIC of $12-40 \mathrm{mg} \mathrm{L}^{-1}$ toward E. coli and $18-80 \mathrm{mg} \mathrm{L}^{-1}$ toward $S$. aureus, respectively. The MBC of these Ag@WMSs samples was also measured, indicating a minimum bactericidal concentration of 24-96 $\mathrm{mg} \mathrm{L}^{-1}$ toward E. coli and $36-108 \mathrm{mg} \mathrm{L}^{-1}$ toward $S$. aureus, respectively. Comparing the three Ag@WMSs samples, Ag@WMSs-NNN exhibited the best antibacterial and bactericidal properties, probably because the high loading content of Ag NPs. Owing to the high surface amino density of WMSs-NN and the strong coordination ability of $\mathrm{KH}-792$, monodisperse Ag NPs with smaller particle size and better dispersibility was generated on WMSs-NN, and therefore $\mathrm{Ag} @ W M S s-N N$ displayed better antibacterial capacity than Ag@WMSs-N2.

\section{Conflicts of interest}

There are no conflicts to declare.

\section{Acknowledgements}

The financial supports from the National Natural Science Foundation of China (51778369), Natural Science Foundation of Guangdong Province (2017A030313047, 2017A030310324), and Fundamental Research Project of Shenzhen City (JCYJ20160520160830116, JCYJ20160422102541990) are gratefully acknowledged.

\section{Notes and references}

1 M. Moritz and M. Geszke-Moritz, Chem. Eng. J., 2013, 228, 596-613.

2 Z. R. Xu, X. L. Zhao, X. D. Chen, Z. H. Chen and Z. F. Xia, $R S C$ Adv., 2017, 7, 52266-52273.

3 K. P. Rakesh, H. K. Vivek, H. M. Manukumar, C. S. Shantharam, S. N. A. Bukhari, H. L. Qin and M. B. Sridhara, $R S C$ Adv., 2018, 8, 5473-5483.

4 S. Agnihotri, S. Mukherji and S. Mukherji, Nanoscale, 2013, 5, 7328-7340.

5 S. Chernousova and M. Epple, Angew. Chem., Int. Ed., 2013, 52, 1636-1653.

6 G. A. Sotiriou and S. E. Pratsinis, Environ. Sci. Technol., 2010, 44, 5649-5654.

7 A. R. Gliga, S. Skoglund, I. O. Wallinder, B. Fadeel and H. L. Karlsson, Part. Fibre Toxicol., 2014, 11, 11.

8 D. Y. Zhao, J. L. Feng, Q. S. Huo, N. Melosh, G. H. Fredrickson, B. F. Chmelka and G. D. Stucky, Science, 1998, 279, 548-552.
9 J. Li, L. Xu, H. Y. Wang, B. X. Yang, H. Z. Liu, W. S. Pan and S. M. Li, Mater. Sci. Eng., C, 2016, 59, 710-716.

10 Z. F. Tian, Y. Xu and Y. F. Zhu, Mater. Sci. Eng., C, 2017, 71, 452-459.

11 X. S. Zhao, X. Y. Bao, W. P. Guo and F. Y. Lee, Mater. Today, 2006, 9, 32-39.

12 F. Jiao and H. Frei, Angew. Chem., Int. Ed., 2009, 48, 18411844.

13 X. C. Li, W. J. Zheng, B. Chen, L. Wang and G. H. He, ACS Sustainable Chem. Eng., 2016, 4, 2780-2788.

14 S. Chatterjee and A. R. Paital, Adv. Funct. Mater., 2018, 28, 1704726.

15 M. Okamoto, H. Satake and H. Seki, J. Mater. Chem. A, 2017, 5, 24425-24432.

16 S. Y. Tan, C. Teh, C. Y. Ang, M. H. Li, P. Z. Li, V. Korzh and Y. L. Zhao, Nanoscale, 2017, 9, 2253-2261.

17 R. Gupta, P. K. Rastogi, V. Ganesan, D. K. Yadav and P. K. Sonkar, Sensor Actuat B-Chem, 2017, 239, 970-978.

18 R. Gupta, P. K. Rastogi, U. Srivastava, V. Ganesan, P. K. Sonkar and D. K. Yadav, RSC Adv., 2016, 6, 6577965788.

19 X. J. Wan, G. Y. Zhang and S. Y. Liu, Macromol. Rapid Commun., 2011, 32, 1082-1089.

20 X. J. Wan, T. Liu, J. M. Hu and S. Y. Liu, Macromol. Rapid Commun., 2013, 34, 341-347.

21 H. Yang, Y. Liu, Q. H. Shen, L. F. Chen, W. H. You, X. M. Wang and J. S. Sheng, J. Mater. Chem., 2012, 22, 24132-24138.

22 Y. Tian, J. J. Qi, W. Zhang, Q. Cai and X. Y. Jiang, ACS Appl. Mater. Interfaces, 2014, 6, 12038-12045.

23 H. Yu, Y. Zhu, H. Yang, K. Nakanishi, K. Kanamori and X. Z. Guo, Dalton Trans., 2014, 43, 12648-12656.

24 C. W. Wang, H. Hong, Z. F. Lin, Y. Yuan, C. S. Liu, X. Y. Ma and X. Y. Cao, RSC Adv., 2015, 5, 104283-104292.

25 D. S. Moon and J. K. Lee, Langmuir, 2012, 28, 12341-12347.

26 L. P. Li, J. Song, J. G. Wang and W. B. Fan, Mater. Express, 2017, 7, 283-290.

27 X. J. Wan, L. L. Zhuang, B. X. She, Y. M. Deng, D. Z. Chen and J. N. Tang, Mater. Sci. Eng., C, 2016, 65, 323-330.

28 T. Yokoi, H. Yoshitake and T. Tatsumi, J. Mater. Chem., 2004, 14, 951-957.

29 Z. P. Dong, X. D. Le, X. L. Li, W. Zhang, C. X. Dong and J. T. Ma, Appl. Catal., B, 2014, 158, 129-135.

30 D. Tian, G. P. Yong, Y. Dai, X. Y. Yan and S. M. Liu, Catal. Lett., 2009, 130, 211-216.

31 S. Egger, R. P. Lehmann, M. J. Height, M. J. Loessner and M. Schuppler, Appl. Environ. Microbiol., 2009, 75, 2973-2976.

32 Y. H. Kim, D. K. Lee, H. G. Cha, C. W. Kim and Y. S. Kang, J. Phys. Chem. C, 2007, 111, 3629-3635.

33 S. Agnihotri, S. Mukherji and S. Mukherji, RSC Adv., 2014, 4, 3974-3983. 